\title{
ON THE POSSIBILITY OF DIRECTLY ACCESSING EVERY HUMAN BRAIN BY ELECTROMAGNETIC INDUCTION OF FUNDAMENTAL ALGORITHMS
}

\author{
By Professor Michael A. Persinger \\ Laurentian University, Ontario
}

June 1995

Perceptual and Motor Skills, June 1995, 80, 791-799 ISSN 0031-5125

[This statement by the author of the following paper says it all:

"Within the last two decades (Persinger, Ludwig, \&

Ossenkopp,1973) a potential has emerged which was improbable

but which is now marginally feasible. This potential is the

technical capability to influence directly the major portion of the

approximately six billion brains of the human species through

classical sensory modalities by generating neural information

within a physical medium within which all members of the species

are immersed."

The medium that he is referring to, is the atmosphere of this planet]

Summary:-- Contemporary neuroscience suggests the existence of fundamental algorithms by which all sensory transduction is translated into the intrinsic, brain-specific code. Direct stimulation of these codes within the human temporal or limbic cortices by applied electromagnetic patterns may require energy levels which are within the range of both geomagnetic activity and contemporary communication networks. A process which is coupled to the narrow band of brain temperature could allow all normal human brains to be affected by a subharmonic whose frequency range at about $10 \mathrm{~Hz}$ would only vary by $0.1 \mathrm{~Hz}$.

The pursuit of the basic algorithms by which all human brains operate can be considered a central theme of modern neuroscience. Although individual differences are expected to accommodate most of the variance in any specific neurobehavioral measure, there should exist basic patterns of information and structure within brain space. They would be determined by the human genome, i.e., be species-specific, and would contribute to or would serve as the substrate upon which all phenomena that affect neurobehavioral measures are superimposed.

One logical extrapolation to a neuro-physical basis of consciousness is that all experiences must exist as correlates of complex but determined sequences of electromagnetic matrices. They would control the theme for the format of cognition and affect while the myriad of possible serial collections of random variations of "noise" within the matrices could potentially differentiate between individual brains. Identification of these sequences could also allow direct access to the most complex neuro-cognitive processes associated with the sense of self, human consciousness and the aggregate of experiential representations (episodic memory) that define the individual within the brain (Squire, 1987).

The existence of fundamental commonalities between all human brains by which a similar physical stimulus can affect them is not a new concept. It is demonstrated daily by the similar shifts in qualitative functions that are evoked by psychotropic drugs. Classes of chemical structures, crudely classified as antidepressant, antipsychotic, or anxiolytic compounds, produce general attenuations of lowered mood, extreme eccentric thinking, or extreme 
vigilance. The characteristics of these changes are very similar within millions of different human brains regardless of their cultural or genetic history. The idiosyncratic experiences such as the specific thoughts and images that reflect each person's continuing process of adaptation are superimposed upon these general functions. When translated into the language of neuroelectrical domains, the unique components of individual consciousness would be both embedded within and interacting with the species-invariant patterns.

We have been studying the phenomenological consequences of exposure to complex electromagnetic fields whose temporal structures have been derived from the most recently observed neuro-electrical profiles such as burst-firing or long-term potentiating sequences (Brown, Chapman, Kairiss, \& Keenan, 1988) which can be considered the prototypical basis of a major domain of brain activity. These temporal patterns of potential codes for accessing and influencing neuronal aggregates have been applied across the two cerebral hemispheres (through the regions of the temporoparietal lobes or within the region of the hippocampalamygdaloid complex) of the brain as weak electromagnetic fields whose intensities are usually less than 10 milligauss (1 microT). The purpose of this research, as suggested by both E.R. John (1967) and Sommerhoff (1974), is to identify the basic codes for the language of the representational systems within the human brain.

In the tradition of Johannes Mueller, we have assumed that the normal transduction of stimuli by sensors into afferent, graded potentials and the subsequent translation into digital patterns of action potentials (which are more likely to behave functionally as a composite of pixels within a neural field) can be circumvented by direct introduction of this information within the brain. Induction of complex information would require simulation of the resonance patterns which would normally be transiently created by sensory afferents. The basic premise is that synthetic duplication of the neuro-electrical correlates generated by sensors to an actual stimulus should produce identical experiences without the presence of that stimulus.

We have focused upon the polymodal and most labile portions of the parahippocampal (Van Hoesen, 1982) and entorhinal cortices (Vinagradova, 1975) and the anterior superior gyrus of the temporal cortices (Bancaud, Brunet-Bourgin, Chauvel, \& Halgren, 1994) as the region within which circumvention would be most probable. Extraction and translation of neural patterns from different sensory inputs into common codes occur within these regions before they are consciously perceived (Edelman, 1989). That central codes are present was shown by E.R. John (1967, pp. 348-349) who reported an immediate transference of the operant control of a response from a pulsatile auditory stimulus to a pulsatile visual stimulus if its temporal pattern was identical to the previous (acoustic) stimulus.

We (Fleming, Persinger, \& Koren, 1994) reported that whole brain exposure of rats to a 5microT burst-firing magnetic field for $1 \mathrm{sec}$. every $4 \mathrm{sec}$. evoked an analgesic response that was similar to that elicited by the application of more noxious, tactile simulation for $1 \mathrm{sec}$. every $4 \mathrm{sec}$. directly to the footpads. Direct electrical stimulation of the limbic structures which simulate episodic, systemic application of muscarinic (cholinergic) agents can evoke electrical kindling (Cain, 1989). More recently, direct induction of chaotic electrical sequences within the labile CA1 region of the hippocampus has been shown either to promote and attenuate paroxysmal discharges (Schiff, Jerger, Duong, Chang, Spano, \& Ditto, 1994).

These results strongly indicate that imitation of the temporal pattern of sensory transmission directly within the brain by any nonbiogenic stimuli can evoke changes which are just as effective as (and perhaps require less energy than) classical transduction. As stated more 
recently and succinctly by E.R. John (1990), the fundamental operation of brain electrical activity suggests that some form of frequency encoding may play a significant role in informational transactions within and between brain structures. Consciousness would be associated with an electromagnetic pattern generated by a neural aggregate with invariant statistical features which are independent of the cells contributing to each feature (John 1990, p. 53).

The effects of applied time-varying magnetic fields upon brain activity have been considered minimal or within the range of normal biological limits unless the intensity of the field exceeded natural endogenous or exogenous (ambient) levels by several orders of magnitude. Until very recently, almost all of the studies from which this conclusion was derived involved highly redundant stimuli such as $60 \mathrm{~Hz}$ fields or repetitive pulses. A simple illustration presents the problem: only $1 \mathrm{~min}$. of a $60-\mathrm{Hz}$ sine-wave field exposes a neural net to 3,600 presentations (60 sec. $x 60$ cycles per sec.) of the same redundant information. Even general estimates of habituation (Persinger, 1979) such as the equation H=IRT2/Rt (IRT=inter-response time, $\mathrm{Rt}=$ duration of response) indicate that habituation to the stimulus would have occurred long before its termination after $1 \mathrm{~min}$. Although the burst-firing frequencies (100 to $200 \mathrm{~Hz}$ ) of the hippocampal neurons, for example, exceed this pattern, they are not temporally symmetrical and exhibit a variability of interstimulus intervals that would contain different information and would attenuate habituation.

The apparent dependence of organismic responses upon the intensity of the applied electromagnetic field, the "intensity-dependent response curve," could simply be an artifact of the absence of biorelevant information within the wave pattern. If the temporal structure of the applied electromagnetic field contained detailed and biorelevant information (Richards, Persinger, \& Koren, 1993), then the intensity of the field required to elicit a response could be several orders of magnitude below the values which have been previously found to elicit changes. For example, Sandyk (1992) and Jacobson (1994) have found that complex magnetic fields with variable interstimulus pulse durations could evoke unprecedented changes in melatonin levels even with intensities within the nanoT range.

The classical counterargument that "very strong" magnetic fields must be present "to exceed or to compensate for the electromagnetic noise associated with intrinsic (Boltzmann) thermal energies" is based upon equations and calculations for the quantitative indices of aggregates of molecular activity and not upon the pattern of their interaction. There are other possibilities. For example, Weaver and Astumian (1990) have shown mathematically that detection of very weak (microV/cm) fields can occur if the response is exhibited within a narrow band of frequencies; the detection is a function of both thermally induced fluctuations in membrane potential and the maximum increment of change in the membrane potential which is evoked by the applied magnetic field. The ion-cyclotron-resonance model which was initiated by the research of Blackman, Bename, Rabinowitz, House, and Joines (1985) and supported by Lerchl, Reiter, Howes, Honaka, and Stokkan (1991) indicates that, when an alternating magnetic field at a distance (resonance) frequency is superimposed upon a steady-state magnetic field, the movement of calcium and other ions can be facilitated with very small energies. More than 25 years ago, Ludwig (1968) developed a compelling (but hereto ignored) mathematical argument which described the absorption of atmospherics within the brain.

Above these minimal thresholds, the information content of the wave structure becomes essential. The simplest analogy would be the response of a complex neural network such as a human being to sonic energy. If only a $1000-\mathrm{Hz}$ (sine wave) tone were presented, the intensity 
required to evoke a response could well exceed $90 \mathrm{db}$; in this instance the avoidant response would be overt and crude. However, if the structure of the sonic field was modified to exhibit the complex pattern which was equivalent to biorelevant information such as "help me, I am dying," field strengths several orders of magnitude weaker, e.g., $30 \mathrm{db}$, could be sufficient. This single, brief but information-rich stimulus would evoke a response which could recruit every major cognitive domain.

If the information within the structure of the applied magnetic field is a major source of its neurobehavioral effect, then the "intensity-dependent" responses which are interpreted as support for experimental hypotheses of biomagnetic interaction could be both epiphenomenal and artifactual. Such amplification of electromagnetic-field strengths would also increase the intensity of the extremely subtle and almost always ignored subharmonics, ripples, and other temporal anomalies which are superimposed upon or within the primary frequency. These subtle anomalies would be due to the artifacts within the different electronic circuits and components whose similarities are based upon the fidelity of the endpoint (the primary frequency) despite the different geometries employed to produce the endpoint.

If information rather than intensity is important for interaction with the neural network (Jahn \& Dunne, 1987), then these unspecified "background" patterns may be the source of both the experimental effects and the failures of inter-laboratory replications. A concrete example of this problem exists within the putative association between exposure to power $(60 \mathrm{~Hz})$ frequency magnetic fields and certain types of cancer. The existence of these transients, often superimposed upon the fundamental $60-\mathrm{Hz}$ frequency, is still the least considered factor in the attempts to specify the characteristics of the fields which promote aberrant mitosis (Wilson, Stevens, \& Anderson, 1990).

Within the last five years, several researchers have reported that direct and significant effects upon specific neuropatterns can be evoked by extremely weak magnetic fields whose intensities are within the range of normal geomagnetic variations. Sandyk (1992) has discerned significant changes in vulnerable subjects such as patients who were diagnosed with neurological disorders following exposure of short durations to magnetic fields whose strengths are within the $\mathrm{pT}$ to $\mathrm{nT}$ range but whose spatial applications are multifocal (a fascestype structure) and designed to introduce heterogeneous patterns within a very localized brain space. The effective components of the field (which are assumed to be discrete temporal patterns due to the modulation of the frequency and intensity of the electromagnetic fields) are not always obvious; however, the power levels for these amplitudes are similar to those associated with the signals (generated globally by radio and communication systems) within which most human beings are exposed constantly.

The most parsimonious process by which all human brains could be affected would require the immersion of all the approximately 6 billion brains of the human species within the same medium; or a coercive interaction because there was facilitation of a very narrow-band window of vulnerability within each brain.

For the first option, the steady-state or "permanent" component of the earth's magnetic field meets the criterion. The possibility that masses of susceptible people could be influenced during critical conditions by extremely small variations (less than 1\%) of the steady-state amplitude $(50,000 \mathrm{nT})$ of the earth's magnetic field such as during geomagnetic storms (50 to $500 \mathrm{nT}$ ) has been discussed elsewhere (Persinger, 1983). Recent experimental evidence which has shown a threshold in geomagnetic activity of about $20 \mathrm{nT}$ to $30 \mathrm{nT}$ for the report of 
vestibular experiences in human beings and the facilitation of limbic seizures in rodents is consistent with this hypothesis.

The potential for the creation of an aggregate process with gestalt-like properties which reflect the average characteristics of the brains that are maintained with this field and that generate the aggregate has also been developed (Persinger \& Lafreniere, 1977) and has been labelled the "geopsyche." This phenomenon would be analogous to the vectorial characteristics of an electromagnetic field which is induced by current moving through billions of elements such as wires contained within a relative small volume compared to the source. Such gestalts, like fields in general, also affect the elements which contribute to the matrix (Freeman, 1990)

The second option would require access to a very narrow limit of physical properties within which all brains are maintained to generate consciousness and the experience of selfawareness. This factor would be primarily loaded by the variable of brain temperature. Although the relationship between absolute temperature and wavelength is generally clear [an example which can be described by Wien's law and is well documented in astrophysics (Wyatt, 1965)], the implications for access to brain activity have not been explored. The fragile neurocognitive processes that maintain consciousness and the sense of self normally exist between 308[degrees] K and 312[degrees]K (35[degrees]C and 39[degrees]C). The fundamental wavelength associated with this emission is about 10 micrometers which is well within the long infrared wavelength.

However, the ratio of this normal range divided by the absolute temperature for normal brain activity which maintains neurocognitive processes is only about 0.013 (4[degrees]K312 [degrees] K), or $1.3 \%$. If there were a subharmonic pattern in naturally occurring or technically generated magnetic fields which also reflected this ratio, then all brains which were operative within this temperature range could be affected by the harmonic. For example, if 11.3 $\mathrm{Hz}$ were one of these subharmonic electromagnetic frequencies, variations of only $1.3 \%$ of this mean, i.e., $11.3 \mathrm{~Hz}+/$ - [plus or minus] $0.1 \mathrm{~Hz}$, would hypothetically be sufficient to affect the operations of all normal brains. If this "major carrier frequency" contained biorelevant information by being modulated in a meaningful way, then the effective intensities could well be within the natural range for background radiation (microwatts $/ \mathrm{cm} 2$ ) and could be hidden as chaotic components within the electromagnetic noise associated with power generation and use.

One of the major direct prophylactics to the effects of these fields would require alterations in core (brain) temperature such as deep but reversible hypothermia. However, this condition would disrupt the biochemical process upon which neuronal activity and hence consciousness depends. Treatments which precipitate alterations in neural activity, similar to those which are associated with crude hypothermia, would be less disruptive. Specific candidates which affect multiple receptor systems such as clozepine (Clozaril) and acepromazine could be possible pharmacological interventions.

The characteristics of the algorithm for euthermic individuals are likely to be conspicuous (once isolated) but should now be hidden within the synchronous activity which is modified and filtered by aggregates of neurons; and modulated by sensory inputs and intrinsic oscillations (Kepler, Marder, \& Abbott, 1990) before they are crudely measured by electrodes.

Because the fundamental algorithm would be essentially a stable parameter of body temperature, most electrode montages (including monopolar to a nonbrain reference, e.g., ear) 
would cancel or attenuate this index. Effectively, the algorithm would be expressed in a manner similar to descriptors for other aggregate phenomena as a physical constant or as a limited set of these constants. This suggestion is commensurate with the observation that the underlying neuronal networks which coordinate millions of neurons manifest the properties of a (mathematical) strange attractor with a very limited number of degrees of freedom (Lopes, Da Silva, Kamphuis, Van Neerven, \& Pijn, 1990).

The physical chemical evidence for a fundamental process, driven by a narrow limit of biological temperature, has been accumulating. Fixed, oscillatory electromagnetic variations have been shown in vitro for enzymes of the glycolytic pathway (Higgins, Frenkel, Hulme, Lucas, \& Rangazas, 1973) whose narrow band of temperature sensitivity (around 37[degrees]C) is well known. Although these oscillations are often measured as periods (2.5min. cycles), Ruegg (1973) reported a clear temperature dependence of these oscillations within a range of 1 to $20 \mathrm{~Hz}$ between 20[degrees]C and 35[degrees]C in invertebrate muscle.

The most probable brain source which might serve as the primary modulatory of these biochemical oscillators would involve structures within the thalamus (Steriade \& Deschenes, 1984). Neuronal aggregates with surprisingly fixed (within $0.1-\mathrm{Hz}$ ) oscillations are found within this structure and depend primarily upon neurons that require gamma amino butyric acid or GABA (von Krosigk, Bal, \& McCormick, 1993). This inhibitory amino acid is specially derived from the normal, temperature-sensitive degradation of glucose by the GABA shunt (Delorey \& Olsen, 1994).

Within the last two decades (Persinger, Ludwig, \& Ossenkopp, 1973) a potential has emerged which was improbable but which is now marginally feasible. This potential is the technical capability to influence directly the major portion of the approximately six billion brains of the human species through classical sensory modalities by generating neural information within a physical medium within which all members of the species are immersed. The historical emergence of such possibilities, which have ranged from gunpowder to atomic fission, have resulted in major changes in the social evolution that occurred inordinately quickly after the implementation. Reduction of the risk of the inappropriate application of these technologies requires the continued and open discussion of their realistic feasibility and implications within the scientific and public domain. 


\section{REFERENCES}

Bancuad, J., Brunet-Bourgin, F., Chauvel, P., \& Halgren, E. Anatomical origin of deja vu and vivid 'memories' in human temporal lobe epilepsy. "Brain" 1994, 117, 71-90.

Blackman, C.F., Bename, S.G., Rabinowitz, J.R., House, D.E., \& Joines, W.T. A role for the magnetic field in the radiation-induced efflux of ions from brain tissue in vitro. "Bioelectromagnetics", 1985, 6, 327-337.

Brown, T.H., Chapman, P.F., Kairiss, E.W., \& Keenan, C.L. Long-term potentiation. "Science”, 1988, 242, 724-728.

Cain, D.P. Excitatory neurotransmitters in kindling; excitatory amino acid, cholinergic and opiate mechanisms. "Neuroscience and Biobehavioral_Reviews" 1989, 13, 269-276.

Delorey, T.M., \& Olsen, R.W. GABA and glycine. In G.J. Siegel, B.W. Agranoff, R.W. Albers, \& P.B. Molinoff (Eds.), "Basic neurochemistry" (5th ed.) New York: Raven, 1994. Pp. 389-399.

Edelman, G.M. The remembered present: a biological theory of consciousness. New York; Basic Books, 1989.

Fleming, J.L., Persinger, M.A., \& Koren, S.A. One second per four second magnetic pulses elevate nociceptive thresholds: comparisons with opiate receptor compounds in normal and seizer-induced brain damaged rats. "Electro-and Magnetobiology”, 1994, 13, 67-75.

Freeman, W.J. On the fallacy of assigning an origin to consciousness. In E.R. John (Ed.), Machinery of the Mind. Boston, MA: Birkhauser, 1990. Pp. 14-26.

Higgins, J., Frenkel, R., Hulme, E., Lucas, A., \& Rangazas, G. The control theoretic approach to an analysis of glycolytic oscillators. In B. Chance, E.K. Pye, A.K. Ghosh, \& B. Hess (Eds.), Biological and biochemical oscillators. New York: Academic Press, 1973. Pp. 127-175.

Jacobson, J.I. Pineal-hypothalamic tract mediation of picoTesla magnetic fields in the treatment of neurological disorders. FASEB Journal , 1994, 8, A656.

Jahn, R.G., \& Dunne, B.J. Margins of reality: the role of consciousness in the physical world. New York: Harcourt, Brace \& Jovanovitch, 1987.

John, E.R. Mechanisms of memory. New York: Academic Press, 1967.

John, E.R. Representations of information in the brain. In E.R. John (Ed.). Machinery of the mind. Boston MA: Birkhauser, 1990. Pp. 27-56. 
Kepler, T.B., Marder, E., \& Abbott, L.F. The effect of electrical coupling on the frequency of model neuronal oscillators. Science, 1990, 248, 83-85.

Krosigk, M. von, Bal, T., \& McCormick, D.A. Cellular mechanisms of a synchronized oscillation in the thalamus. Science, 1993, 261, 361-364.

Lerchl, A., Reiter R.J., Howes, K.A., Honaka, K.O., \& Stokkan, K-A. Evidence that extremely low frequency $\mathrm{Ca}++$ cyclotron resonance depresses pineal melatonin synthesis in vitro. Neuroscience Letters, 1991, 124, 213-215.

Lopes, F.H., Da Silva, L., Kamphuis, W., Van Neerven, J.M.A.M., \& Pijn, P.M.Cellular and network mechanisms in the kindling model of epilepsy: the role of GABAnergic inhibition and the emergence of strange attractors. In E.R. John (Ed.), Machinery of the mind. Boston, MA: Birkhauser, 1990. Pp. 115-139.

Ludwig, H.W. A hypothesis concerning the absorption mechanism of atmospherics in the nervous system. International Journal of Biometeorology, 1968, 12, 93-98.

Persinger, M.A. A first approximation of satiation time: (IRT2/Rt). Perceptual and Motor Skills, 1979, 49, 649-650.

Persinger, M.A. The effects of transient and intense geomagnetic or related global perturbations upon human group behavior. In J.B. Calhoun (Ed.), Perspectives on adaptation, environment and population. New York: Praeger, 1983. Pp. 28-30.

Persinger, M.A., \& Lafreniere, G.F. Space-time transients and unusual events. Chicago, IL: Nelson-Hall, 1977.

Persinger, M.A., Ludwig, H.W. \& Ossenkopp, K.P. Psychophysiological effects of extremely low frequency electromagnetic fields: a review. Perceptual and Motor Skills, 1973, 36, 1131-1159.

Richards, P.M., Persinger, M.A., \& Koren, S.A. Modification of activation and evaluation properties of narratives by weak complex magnetic field patterns that simulate limbic burst firing. International Journal of Neuroscience, 1993, 71, 7185.

Ruegg, J.C. Oscillating contractile structures from insect fibrillar muscle. In B. Chance, E.K. Pye, A.K. Ghosh, \& B. Hess (Eds.), Biological and biochemical oscillators. New York: Academic Press, 1973. Pp. 303-309.

Sandyk, R. Successful treatment of multiple sclerosis with magnetic fields. International Journal of Neuroscience, 1992, 66, 237-250.

Schiff, S.J., Jerger, D.H., Chang, T., Spano, M.L., \& Ditto, W.L. Controlling chaos in the brain. Nature, 1994, 370, 615-620.

Sommerhoff, G. Logic of the living brain. New York: Wiley, 1974.

Squire, L.R. Memory and the brain. New York: Oxford Univer. Press, 1987. 
Steriade, M., \& Deschenes, M. The thalamus as a neuronal oscillator. Brain Research Reviews, 1984, 8, 1-63.

Van Hoesen, G.W. The parahippocampal gyrus: new observations regarding its cortical connections in the monkey. Trends in the Neurosciences, 1982, 5, 340-345.

Vinogradova, O.S. Functional organization of the limbic system in the process of registration of information: facts and hypotheses. In R.L. Isaacson \& K.H. Pribram (Eds.), The hippocampus: Vol. 2. Neurophysiology and behavior. New York: Plenum, 1975. Pp. 3-69.

Weaver, J.C., \& Astumian, R.D. The response of living cells to very weak electric fields: the thermal noise limit. Science, 1990, 247, 459-462.

Wilson, B.W. Stevens, R.G., \& Anderson, L.E. Extremely low frequency electromagnetic fields: the question of cancer. Richland, WA: Battelle Press, 1990.

Wyatt, S.P. Principles of astronomy. Boston, MA: Allyn \& Bacon, 1965.

*Please send reprint requests and correspondence to Dr. M.A. Persinger, Behavioral Neuroscience Laboratory, Laurentian, Ramsey Lake Road, Sudbury, Ontario P3E 2C6, Canada.

[Personal background on Professor Persinger and his work, from the Laurentian University website at http://laurentian.ca/neurosci/_people/Persinger.htm follows]

\section{Dr.Michael Persinger}

I was born 26 June 1945 in Jacksonville Florida. I was reared primarily in Virginia, Maryland and Wisconsin. After attending Carroll College (1963-1964), I was graduated from the University of Wisconsin, Madison (1967). Psychology ("Psychochemistry") was selected as a major because it was the interface between the social and physical sciences. I obtained my M.A. (Physiological Psychology) from the University of Tennessee and my Ph.D. from the University of Manitoba (1971). I have been employed as a professor at Laurentian University in Sudbury, Ontario Canada since 1971. During this period I have published more than 300 technical articles in referred journals and have written six books (see full C.V).

My primary philosophical goal is to discern the commonalities that exist between the sciences and to integrate the fundamental concepts. I assume that the human brain, its microstructure and intricate activity are the source of all human knowledge. To that end I have emphasised geophysics because it is a central focus for the physical sciences and neuroscience (originally physiological psychology) because it is a central focus for the emerging biosocial sciences. One of the major consequences of this bilateral interest has been the pursuit and discovery of subtle interactions between the geophysical/ meteorological environment and human behavior. 
Because scientific explanations and attributions are transient labels applied to the largely inferred and unseen shared sources of variance within numerical data (or verbal responses that serve as nominal data), I have pursued methodology and multivariate (statistical) approaches. Magnetic fields were selected as a focus because they are one of the few stimuli that evoke changes across all levels of scientific discourse. This perspective was summarized in ELF and VLF Electromagnetic Field Effects (1974) and Space-Time Transients and Unusual Events (1977). These approaches in conjunction with the goal of integrating concepts have influenced my decision to investigate interdisciplinary problems and to apply these skills both within academic and practical settings.

Within academic settings, I organized the Behavioral Neuroscience Program at Laurentian University. This program was one of the first to integrate Chemistry, Biology and Psychology. The program was developed because there is a subset of students with integrative capacity who are not "A" students but who are extraordinary problem solvers who love to learn. Within clinical settings, I became a Registered Psychologist, specializing in Clinical Neuropsychology, in order to facilitate the integration of neurology, neuropsychology and psychology and to develop quantitative methods whose results could help facilitate the adaptation of people who have sustained mild to moderate brain traumas. Within the commercial setting, we have pursued the possibility that control of experience, from depression to memory, may be simulated by transcerebral application of complex magnetic field patterns associated with activity of either endogenous or exogenous ligands at the synapses.

As a human being, I am concerned about the illusionary explanations for human consciousness and the future of human existence. Consequently after writing the Neuropsychological Base of God Beliefs (1987), I began the systematic application of complex electromagnetic fields to discern the patterns that will induce experiences (sensed presence) that are attributed to the myriad of ego-alien intrusions which range from gods to aliens. The research is not to demean anyone's religious/mystical experience but instead to determine which portions of the brain or its electromagnetic patterns generate the experience. Two thousand years of philosophy have taught us that attempting to prove or disprove realities may never have discrete verbal (linguistic) solutions because of the limitation of this measurement. The research has been encouraged by the historical fact that most wars and group degradations are coupled implicitly to god beliefs and to the presumption that those who do not believe the same as the experient are somehow less human and hence expendable. Although these egocentric propensities may have had adaptive significance, their utility for the species' future may be questionable.

Because our work is interdisciplinary and may be different from what others have done, I insist that the techniques and results are published within the public forum (the scientific literature). This procedure will hopefully decrease the probability that the technology or knowledge will be employed only by a privileged few. Except for $\$ 10,000$ given to us in 1983 by a researcher (from the U.S. Navy) who was interested in magnetic fields and brain activity, all of my work has been supported out of my pocket primarily from my private practice. Laurentian University has been consistently supportive by supplying space and infrastructure. We have been fortunate that the topics of research our laboratory have preceded the interests of the scientific social community by about 10 to 15 years. 